\title{
EFFECT OF BOUNDARY CONDITIONS ON THE HYDRODYNAMICS AND HEAT TRANSFER IN FLUIDIZED BEDS SIMULATIONS
}

\author{
P. C. BISOGNIN ${ }^{1}$, J. M. FUSCO ${ }^{2}$ e C. SOARES ${ }^{1}$ \\ ${ }^{1}$ Federal University of Santa Catarina, Department of Chemical and Food Engineering \\ ${ }^{2}$ State University of Campinas, Department of Chemical Engineering \\ E-mail contact: cintia.soares@ufsc.br
}

\begin{abstract}
Due to its great industrial application, fluidized beds have been largely studied. With the increase in computational power, researchers started to study these equipments computationally, through CFD (Computational fluid dynamics). This work has the objective to improve fluidized bed simulations by defining the best value of the specularity coefficient, an important parameter related to the boundary condition of the wall. It was found that this parameter not only influences the hydrodynamics of the bed but also the heat transfer coefficient between an immersed heated surface and the bed. Six different specularity coefficients were tested and the results were compared to experimental data. Further studies need to be made in order to verify if the results encountered in this work can be applied in other fluidization states and bed geometries and how other simulation parameters could alter these results.
\end{abstract}

\section{INTRODUCTION}

Fluidized beds are extensively used in chemical, petrochemical, pharmaceutical and mineral industries. Because of their complex hydrodynamics most of the developing and designing of fluidized beds has been empiric (Taghipour et al., 2005). With the increase of the computational capacity, CFD (Computational Fluid Dynamics) has proved itself a powerful tool to study different hydrodynamic systems. Nowadays, the use of the Eulerian approach and twofluid models (TFM), which consider the two phases as interpenetrating continua, to simulate fluidized beds is a common practice (Acosta-Iborra et al., 2011). Most part of the chemical reactions requires either heat input or extraction. For that reason the understanding not only of the hydrodynamics, but also of the mechanisms of heat transfer inside fluidized beds is fundamental (Shi et al., 1998).

Several works have discussed the gas-solid interaction, in terms of the drag correlation and the collision and frictional interactions between particles, but less attention has been given to the interaction between the particles and the wall (Lan et al., 2012). Li et al. (2010b) concluded that the boundary conditions for the gas phase need to be well specified when gas-mixing is modeled after they tested free-slip, no-slip and partial-slip boundary condition on the wall and obtained substantially different results. The Johnson and Jackson (1987) wall boundary condition is 
largely applied. It includes two parameters, the specularity coefficient, $\varphi$, that specifies the shear condition at the wall, and the particle-wall restitution coefficient, $e_{p w}$, that represents the dissipation of the solid kinetic energy with the walls by collisions (Zhong et al., 2012). For $\varphi=0$ a free-slip condition is applied, while for $\varphi=1$ a no-slip boundary condition is employed.

Recently researchers started to investigate the importance of the boundary conditions in fluidized beds simulations using CFD. Kong et al. (2013) used different values of specularity coefficient and restitution coefficient to simulate circulating fluidized beds (CFB) and attested the importance of this parameter in these simulations. Zhou et al. (2013) studied the wall boundary conditions in CFB simulations and concluded that the influence of the specularity coefficient on the solids distribution depends on the drag model. Lan et al. (2012) concluded that the specularity coefficient has a large influence on the predicted behavior of spouted beds. Li et al. (2010a) studied the wall boundary conditions in bubbling fluidized beds simulations and concluded that the wall effects have an important role on CFD models.

Based on these statements the aim of this work is to show how different specularity coefficients can affect the hydrodynamic of a fluidized bed and to define the best value of specularity coefficient to be used in heat transfer problems.

\section{NUMERICAL SIMULATION}

\subsection{Numerical setup}

All simulations were carried out with a two-dimensional geometry based on the experimental work of Di Natale et al. (2007). The width of the bed was $0.1 \mathrm{~m}$ and the height was $1.8 \mathrm{~m}$. Initially the height of $0.6 \mathrm{~m}$ of the bed was filled with particles with diameter of $500 \mu \mathrm{m}$. The inlet gas velocity was $0.45 \mathrm{~m} / \mathrm{s}$. Inside the bed there was a heated cylinder (diameter $20 \mathrm{~mm}$, height $30 \mathrm{~mm}$ ). Figure 1 shows the geometry used, considering that gravity is acting in the horizontal axis. This is a simplified geometry, in future studies a new geometry containing the cylinder support will be created.

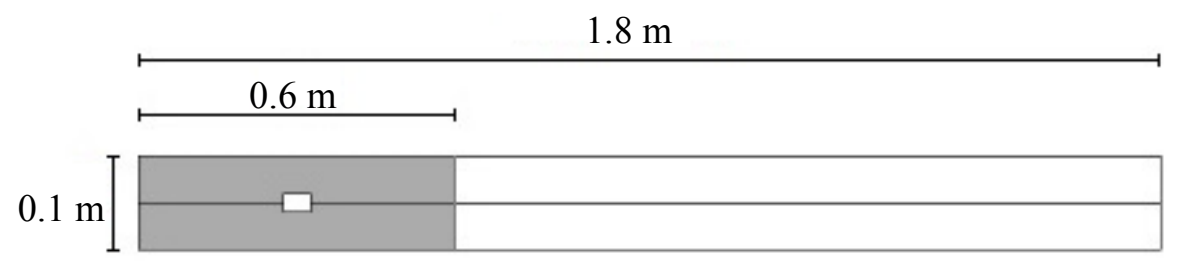

Figure 1 - Two-dimensional geometry used in the simulations*.

\subsection{Governing Equations}

In the Euler-Euler two-fluid model the conservation equations for the solid and gas phase are solved individually and closed with the appropriate constitutive equations. The continuity equations for the gas (g) and solid (s) phases can be written as: 


$$
\begin{aligned}
& \frac{\partial}{\partial t}\left(\alpha_{g} \rho_{g}\right)+\nabla \cdot\left(\alpha_{g} \rho_{g} \vec{v}_{g}\right)=0 \\
& \frac{\partial}{\partial t}\left(\alpha_{s} \rho_{s}\right)+\nabla \cdot\left(\alpha_{s} \rho_{s} \vec{v}_{s}\right)=0,
\end{aligned}
$$

where $\alpha$ is the volume fraction, $\rho$ is the density and $\vec{v}_{s}$ is the velocity vector.

The momentum equations for the gas and solid phases are given as follow:

$$
\begin{aligned}
& \frac{\partial}{\partial t}\left(\alpha_{g} \rho_{g} \vec{v}_{g}\right)+\nabla \cdot\left(\alpha_{g} \rho_{g} \vec{v}_{g} \vec{v}_{g}\right)=-\alpha_{g} \nabla p+\nabla \cdot \overline{\bar{\tau}}_{g}+\alpha_{g} \rho_{g} g+K_{g s}\left(\vec{v}_{s}-\vec{v}_{g}\right) \\
& \frac{\partial}{\partial t}\left(\alpha_{s} \rho_{s} \vec{v}_{s}\right)+\nabla \cdot\left(\alpha_{s} \rho_{s} \vec{v}_{s} \vec{v}_{s}\right)=-\alpha_{s} \nabla p-\nabla p_{s}+\nabla \cdot \overline{\bar{\tau}}_{s}+\alpha_{s} \rho_{s} g+K_{g s}\left(\vec{v}_{g}-\vec{v}_{s}\right),
\end{aligned}
$$

where $p$ is the hydrodynamic pressure, $\overline{\bar{\tau}}_{s}$ is the viscous stress tensor, $g$ is the acceleration due to gravity, $K_{g s}$ is the momentum transfer between gas and solid phases and $p_{s}$ is the solid-phase pressure. The stress tensor of the gas and solid phases are written as follows:

$$
\begin{aligned}
& \overline{\bar{\tau}}_{g}=\alpha_{g} \mu_{g}\left(\nabla \vec{v}_{g}+\nabla \vec{v}_{g}^{T}\right)-\frac{2}{3} \alpha_{g} \mu_{g}\left(\nabla \cdot \vec{v}_{g}\right) \overline{\bar{I}} \\
& \overline{\bar{\tau}}_{s}=\alpha_{s} \mu_{s}\left(\nabla \vec{v}_{s}+\nabla \vec{v}_{s}^{T}\right)-\alpha_{s}\left(\lambda_{s}-\frac{2}{3} \mu_{s}\right)\left(\nabla \cdot \vec{v}_{s}\right) \overline{\bar{I}},
\end{aligned}
$$

where $\mu_{s}$ is the solid shear viscosity and $\lambda_{s}$ is the solid bulk viscosity.

The shear force at the wall for the granular phase is given by:

$$
\overline{\tau_{s}}=\frac{\pi}{6} \sqrt{3} \varphi \frac{\alpha_{s}}{\alpha_{s, \max }} \rho_{s} g_{0} \overline{U_{S, \|}} \sqrt{\Theta_{s}},
$$

where $\overline{U_{S, \|}}$ is the particle slip velocity parallel to the wall, $\varphi$ is the specularity coefficient, $\Theta_{\mathrm{s}}$ is the granular temperature, $g_{0}$ is the radial distribution function and $\alpha_{\mathrm{s} \text {, max }}$ is the volume fraction for the particles at the maximum packing.

* The figure was presented horizontally due to space limitations. 
The thermal energy balance equations for both phases represent the energy accumulation due to convection, diffusion and interphase heat transfer:

$$
\begin{aligned}
& \frac{\partial}{\partial t}\left(\alpha_{g} \rho_{g} H_{g}\right)+\frac{\partial}{\partial x_{i}}\left(\alpha_{g} \rho_{g} U_{i, g} H_{g}\right)=\frac{\partial}{\partial x_{i}}\left(\alpha_{g} k_{g} \frac{\partial T_{g}}{\partial x_{i}}\right)+\gamma_{v}\left(T_{s}-T_{g}\right) \\
& \frac{\partial}{\partial t}\left(\alpha_{s} \rho_{s} H_{s}\right)+\frac{\partial}{\partial x_{i}}\left(\alpha_{s} \rho_{s} U_{i, s} H_{s}\right)=\frac{\partial}{\partial x_{i}}\left(\alpha_{s} k_{s} \frac{\partial T_{s}}{\partial x_{i}}\right)+\gamma_{v}\left(T_{g}-T_{s}\right),
\end{aligned}
$$

where $\mathrm{k}$ is the thermal conductivity, $\mathrm{H}$ is the enthalpy, $\gamma_{\mathrm{v}}$ is the interphase volumetric heat transfer coefficient and $\mathrm{T}_{\mathrm{g}}$ and $\mathrm{T}_{\mathrm{s}}$ are the gas and solids temperature, respectively.

The heat transfer model used in this work is given by Gunn (1978) in terms of the Prandtl number and the particle Reynolds number:

$$
\begin{gathered}
N u_{s}=\left(7-10 \alpha_{g}+5 \alpha_{g}^{2}\right)\left(1+0.7\left(\operatorname{Re}_{p}\right)^{0.2}(\operatorname{Pr})^{1 / 3}\right)+ \\
\left(1.33-2.40 \alpha_{g}+1.20 \alpha_{g}^{2}\right)\left(\operatorname{Re}_{p}\right)^{0.2}(\operatorname{Pr})^{1 / 3}
\end{gathered}
$$

where $\operatorname{Pr}$ is the Prandtl number, $\mathrm{Re}_{\mathrm{p}}$ is the particle Reynolds number and $\mathrm{C}_{\mathrm{p}}$ is the specific heat.

In this work the momentum exchange, $K_{g \mathrm{~s}}$, is based on Gidaspow's model (1994) and the viscosity and pressure of the solid phase is obtained with the kinetic theory of granular flow (KTGF) (Gidaspow, 1994). The solid bulk viscosity and solid pressure are given by Lun et al. (1984) and the solid shear viscosity is given by Gidaspow (1994).

\subsection{Initial and Boundary Conditions}

At the inlet a constant gas velocity was set. At the cylinder wall it was defined a constant value of heat flux, according to the work of Di Natale et al. (2007). At the outlet of the bed, the atmospheric boundary condition was applied. At the wall of the cylinder and of the bed a no-slip condition was used for the gas and the slip boundary condition of Johnson and Jackson (1987) was used for the solid phase. In this boundary condition, the specularity coefficient must be specified to characterize the tangential momentum transfer due to collision of the particles on the wall. Six values of specularity coefficient have been tested, $0,0.05,0.1,0.25,0.5$ and 1 , in order to cover the whole range of values and focus on lower values, usually reported in literature for bubbling beds.

Initially the bed was packed with particles to the height of $0.6 \mathrm{~m}$ of the reactor. It was considered that in this configuration the solids volume fraction was 0.64 , according to the work of Kamien and Liu (2007). 


\subsection{Solution Procedure}

The CFD package ANSYS® CFD (FLUENT®), version 15.0 was used to simulate the case described using axissymetry. The axissymetry was chosen because the main parameter being studied is the heat transfer coefficient which represents a local measure and therefore the results differ little from a two-dimensional geometry, besides that, a two-dimensional geometry wouldn't be able to capture the heat dissipation through the circumference of the bed. A first order scheme was used on the discretization of convective terms and the phase-coupled SIMPLE algorithm was used for the pressure-velocity coupling. The simulations were performed for a time of $10 \mathrm{~s}$, while the period between 5 and $10 \mathrm{~s}$ was used to obtain time-averaged results. In order to avoid instabilities, a step size of $2 \cdot 10^{-4}$ was employed and the convergence criterion was set to $1 \cdot 10^{-3}$.

\section{RESULTS}

\subsection{Grid Independence}

To determine the grid to be used in the simulations, seven different grids, with uniform element sizes of $0.01,0.008,0.006,0.005,0.0045,0.004$ and $0.0035 \mathrm{~m}$, were tested. The time averaged distribution of the solids in the bed and the time averaged axial pressure were computed and it was found that nearly independent results can be obtained with an element size of $0.0045 \mathrm{~m}$.

\subsection{Solids Distribution}

The time averaged solids distributions along the bed height were computed for the six different specularity coefficient used in the simulations, as shown in Figure 2. The results were analyzed in the last $5 \mathrm{~s}$ of simulation, to exclude start up effects, and until $1 \mathrm{~m}$ height, the maximum bed expansion.

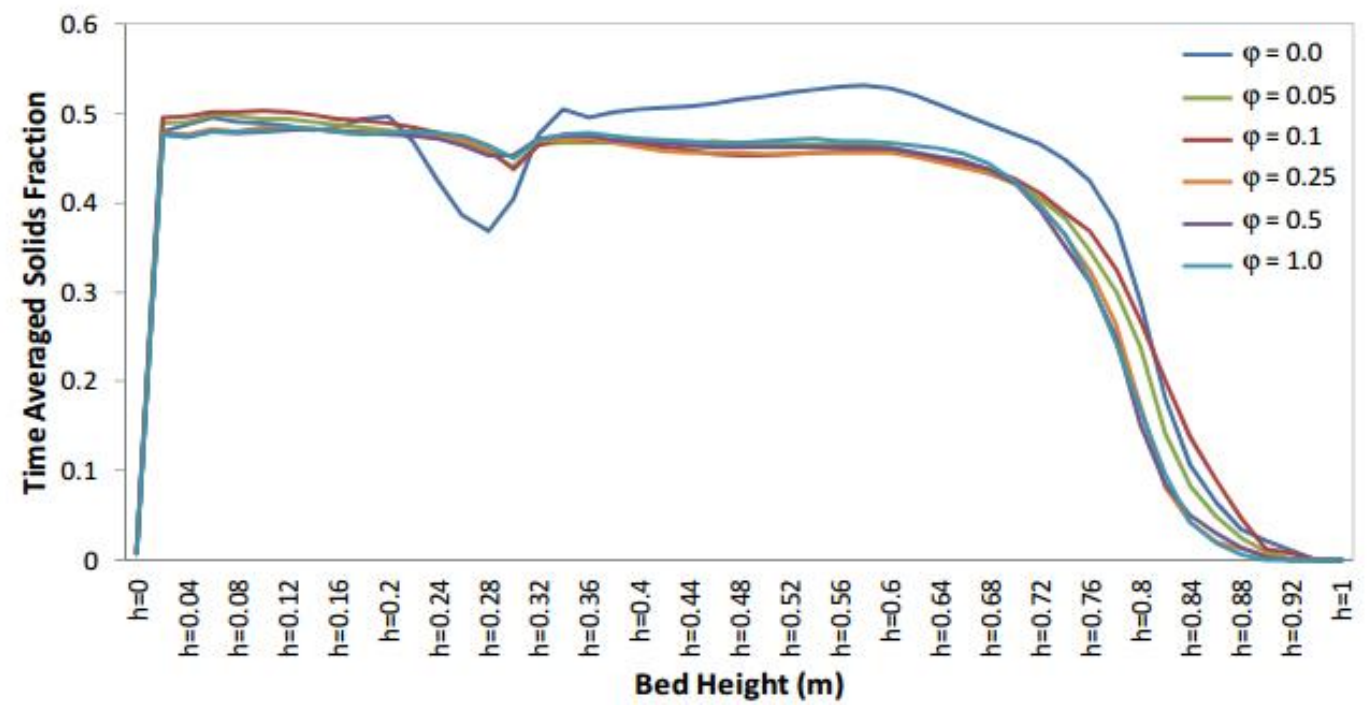

Figure 2 - Time averaged solids fraction distribution for different specularity coefficients. 
It can be seen in Figure 2 the influence of the specularity coefficient on the bed hydrodynamics. The use of $\varphi=0$ produces more pronounced differences in the results, while for the other values the hydrodynamics presents less variation. This little variation on the bed hydrodynamics for values different than zero may explain why most authors use $\varphi=1$ without further testing other values.

Figure 3 shows the solids volume fraction in five different times of the simulation: 5, 6, 7, 8, 9 and $10 \mathrm{~s}$, for four different specularity coefficients. The color red represents a solids volume fraction of 0.64 and the color blue represents the absence of solids. It can be seen that the change in the specularity coefficient alters the behavior of the bed, especially for $\varphi=0$, where the bubbles concentrate at the wall.
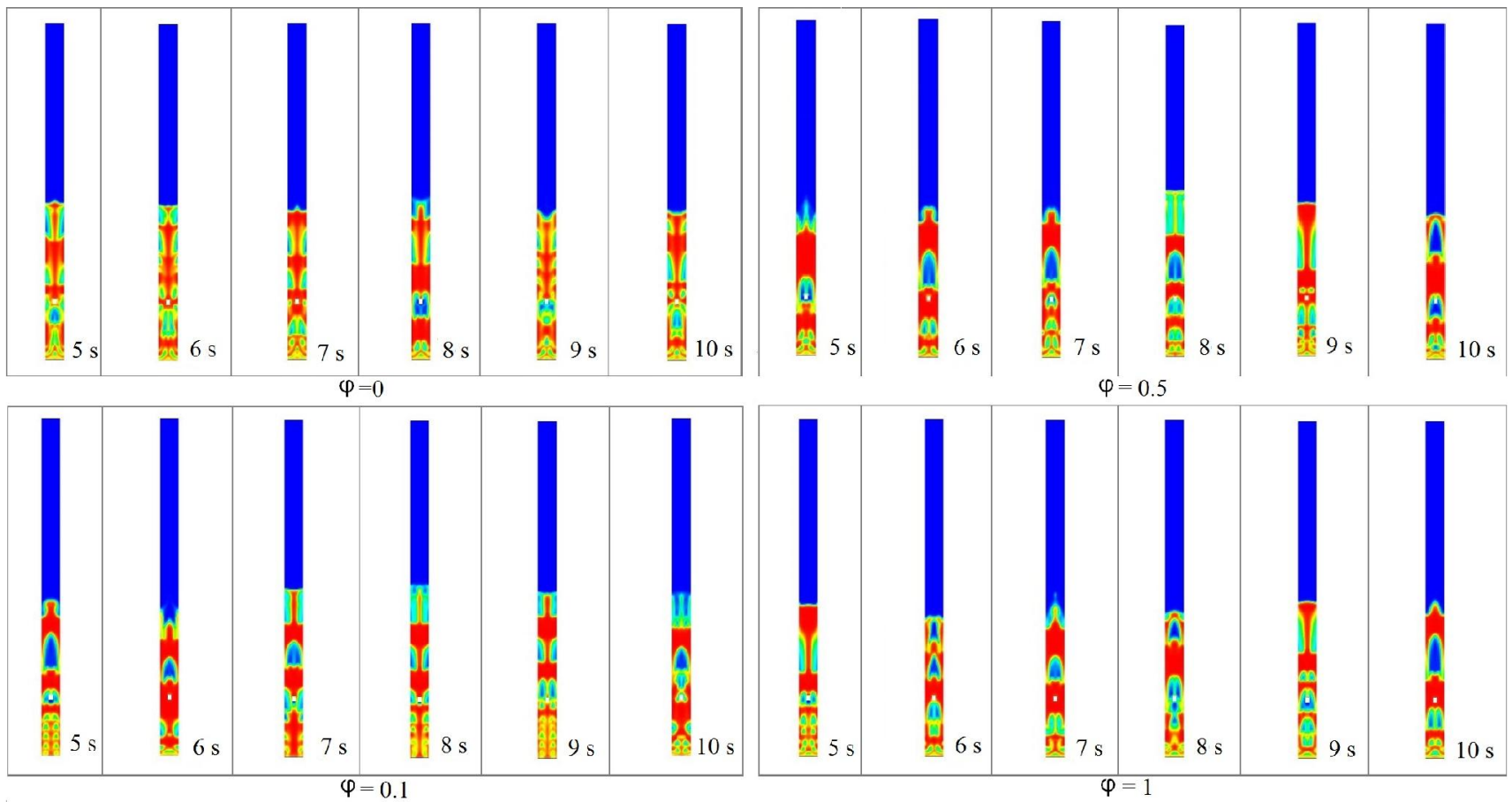

Figure 3 - Solids fraction distribution using different specularity coefficients.

\subsection{Heat Transfer Coefficient}

Although recent papers have been studying the effects of the specularity coefficient, no author have studied the influence of this parameter on the heat transfer coefficient between the bed and a heated surface immersed on the bed. The heat transfer coefficient was calculated in the same point as in Di Natale et al. (2007) in order to compare the obtained results with experimental results. Table 1 brings the time averaged heat transfer coefficient for each specularity coefficient used and Table 2 shows the variation of the simulations results with the experimental result obtained by Di Natale et al. (2007). 
Table 1 - Time averaged heat transfer coefficient $\left(\mathrm{W} / \mathrm{m}^{2} . \mathrm{K}\right)$

\begin{tabular}{cccccc}
\hline$\varphi=0$ & $\varphi=0.05$ & $\varphi=0.1$ & $\varphi=0.25$ & $\varphi=0.5$ & $\varphi=1$ \\
\hline 163.10 & 175.27 & 175.94 & 140.39 & 154.72 & 136.39 \\
\hline
\end{tabular}

Table 2 - Results deviation compared to the experimental data of Di Natale et al. (2007)

\begin{tabular}{cccccc}
\hline$\varphi=0$ & $\varphi=0.05$ & $\varphi=0.1$ & $\varphi=0.25$ & $\varphi=0.5$ & $\varphi=1$ \\
\hline $29.09 \%$ & $23.80 \%$ & $23.51 \%$ & $38.96 \%$ & $32.73 \%$ & $40.70 \%$ \\
\hline
\end{tabular}

Table 2 reveals that even though there is little variation in the bed hydrodynamics, the heat transfer coefficient is altered by the specularity coefficient, with the value of 0.1 presenting the best result for this particular case. It is known that the drag coefficient model plays a major role in fluidized bed simulations and therefore this parameter should be investigate along with the specularity coefficient to better understand the heat transfer process inside the bed. Li et al. (2010b) suggested that the specularity coefficient may be influenced by the superficial gas velocity. New simulations must be made varying different parameters to study their interactions with the wall boundaries in the proposed problem in order to define the optimal specularity coefficient value to study heat transfer in fluidized beds.

\section{CONCLUSIONS}

In the present study it was shown the importance of the right choice of the wall boundary conditions in simulations of fluidized beds. Other authors have already proved the importance of the role played by the specularity coefficient on the hydrodynamics of the bed, but this study also proves its influence on the heat transfer coefficient. There are more pronounced differences in the bed hydrodynamics when a value of 0 is used for the specularity coefficient, with other values presenting a similar behavior. That explains why most authors do not test different specularity coefficient values. However this parameter showed a great influence on the heat transfer coefficient and it was found that for this case the best value for the specularity coefficient is 0.1 . To deeply understand the role of the coefficient specularity in heat transfer problems inside fluidized beds it needs to be studied along with other simulation parameters, such as the drag coefficient model and superficial gas velocity. Future work include the creation of a three-dimensional geometry to better represent fluidization, the use of second order discretization scheme and the study of different drag coefficient and heat transfer models.

\section{ACKNOWLEDGEMENTS}

The authors acknowledge CAPES for the financial support.

\section{REFERENCES}

ACOSTA-IBORRA, A.; SOBRINO, C.; HERNÁNDEZ-JIMÉNEZ F.; VEGA, M. Experimental and computational study on the bubble behavior in a 3-D fluidized bed. Chem. Eng. Sci., v. 66, p. 3499-3512, 2011. 
DI NATALE, F.; LANCIA, A.; NIGRO R. Surface-to-bed heat transfer in fluidised beds: Effect of surface shape, Powder Technol., v. 174, p. 75-81, 2007.

GIDASPOW, D. Multiphase flow and fluidization: Continuum and kinetic theory descriptions. New York: Academic Press, 1994.

JOHNSON, P.; JACKSON, R. Frictional-collisional constitutive relations for granular materials, with application to plane shearing. J. Fluid. Mech., v.176, p. 67-93, 1987.

KAMIEN, R. D.; LIU, A. J. Why is Radom Close Packing Reproducible? Phys. Rev. Lett.,v. 99, p. $1555011-4,2007$.

KONG, L.; ZHANG, C.; ZHU J. Evaluation of the effect of wall boundary conditions on numerical simulations of circulating fluidized beds, Particuology, Available online 8 July 2013, http://dx.doi.org/10.1016/j.partic.2013.04.007.

LAN X.; XU, C.; GAO, J.; AL-DAHHAN, M. Influence of solid-phase wall boundary condition on CFD simulation of spouted beds. Chem. Eng. Sci., v. 69, p. 419-430, 2012

LI, T.; GRACE, J.; BI, X. Study of wall boundary condition in numerical simulations of bubbling fluidized beds. Powder Technol., v. 203, p. 447-457, 2010 a.

LI, T.; ZHANG, Y.; GRACE, J. R.; BI, X. Numerical investigation of gas mixing in gas-solid fluidized beds. AIChE J., v. 56, p. 2280-2296, 2010b.

LUN, C. K. K.; SAVAGE, S. B.; JEFFREY, D. J.; CHEPURNITY, N. Kinetic theories for granular flow: Inelastic particles in Couette flow and slightly inelastic particles in a general flow field. J. Fluid. Mech., v. 140, p. 223-256, 1984.

SHI, D.; NICOLAI R.; REH, L. Wall-to-bed heat transfer in circulating fluidized beds, Chem. Eng. Process, v. 37, p. 287-293, 1998

TAGHIPOUR, F.; ELLIS, N.; WONG, C. Experimental and computational study of gas-solid fluidized bed hydrodynamics. Chem. Eng. Sci., v. 60, p. 6857-6867, 2005.

ZHONG, H.; GAO, J.; XU, C.; LAN, X. CFD modeling the hydrodynamics of binary particle mixtures in bubbling fluidized beds: Effect of wall boundary condition. Powder Technol., v. 230, p. 232-240, 2012.

ZHOU, X.; GAO, J.; XU, C.; LAN, X. Effect of wall boundary condition on CFD simulation of CFB risers. Particuology, v. 11, p. 556-565, 2013. 